\title{
Load-Aware Heterogeneous Cellular Networks: Modeling and SIR Distribution
}

\author{
Harpreet S. Dhillon, Radha Krishna Ganti and Jeffrey G. Andrews
}

\begin{abstract}
Heterogeneous cellular networks (HCNs) are characterized by cells whose coverage areas may vary by orders of magnitude. It is natural therefore that their user populations (and hence traffic loads) will vary similarly. Yet, to date, random spatial models developed for HCNs generally assume that all base stations (BSs) are always transmitting and hence implicitly have the same load. This paper incorporates a flexible notion of BS load by conditionally thinning the interference field, conditional on the connection of a typical mobile to its serving BS. We derive the coverage probability - i.e. the Signal-to-Interference-Ratio (SIR) distribution - for a typical mobile in a K-tier HCN where each tier has an arbitrary load characterized by a traffic factor $p_{k} \in[0,1]$, where $p_{k}=1$ is fully loaded. Fully-loaded models are observed to be extremely pessimistic in terms of coverage, and the analysis shows that adding lightly loaded access points (e.g. pico or femtocells) to the macrocell network always increases the coverage probability.
\end{abstract}

\section{INTRODUCTION}

Due to the increasing popularity of mobile data and video, focus has shifted from voice-oriented applications towards data-hungry applications such as live video streaming and symmetric video calls [1]. Macrocell based conventional cellular networks were primarily designed to provide coverage and are clearly not capable of accommodating this huge change in the usage trends [2]. As a result, a typical $3 \mathrm{G}$ or $4 \mathrm{G}$ cellular network already has microcells, picocells, distributed antennas, and femtocells, along with the existing macrocell BSs.

This rapidly increasing heterogeneity requires new models, e.g., a random spatial model in which the BS locations form a realization of some random spatial point process [3]. Such a model captures the inevitable uncertainty in their locations, and tools from stochastic geometry, point process theory and spatial statistics can be deployed to assist in analysis [4]. This model was introduced for HCNs in [5], [6] and extended in [7]-[9], and is surprisingly tractable: under fairly benign assumptions, the coverage probability could be derived in closed-form, which is not possible even for 1-tier networks in the deterministic hexagonal grid model. The model further was shown to generally agree in several important ways with more sophisticated industry (e.g. 3GPP) simulations [10] and even early field deployments of HCNs [11].

Despite this progress, these (and other) HCN models neglect network traffic and load, instead assuming that all the BSs transmit concurrently all the time. Although this might be

H. S. Dhillon and J. G. Andrews are with the Wireless Networking and Communications Group (WNCG), The University of Texas at Austin, TX, USA (email: dhillon@utexas.edu and jandrews@ece.utexas.edu). R. K. Ganti is with the department of EE at the Indian Institute of Technology Madras, India (email: rganti@ee.iitm.ac.in). justified for macrocells, it is clearly unrealistic for smaller cells with far fewer users, on average. The main goal of this paper is to overcome this shortcoming by incorporating a notion of BS load, while retaining some of the tractability which makes random spatial models, especially Poisson Point Process (PPP), so attractive for analysis. The paper's contributions are now summarized.

Tractable Load Model for $K$-Tier HCNs. The first contribution is the proposed model and framework, which incorporates a simple notion of load. For an HCN where BSs across tiers differ in terms of their transmit power, supported data rate and deployment density, we assume that a typical mobile connects to the strongest BS in terms of received power and conditioned on this connection, the $i^{t h}$-tier interfering BSs transmit independently with a probability $p_{i}$. These BS activity factors $\left\{p_{i}\right\}$ may vary significantly across the tiers due to different coverage areas of each tier.

Coverage Probability. We derive exact expressions for the coverage probability of a typical mobile user in both open and closed access HCNs. Equivalently, this gives the outage probability and characterizes the signal-to-interference-ratio (SIR) distribution over the network.

Design Insights. We observe that adding lightly loaded small cells, such as femto or picocells, to the macrocell network always increases the coverage probability, which is an optimistic result for current cellular trends and counter to the commonly held belief that adding in-band "interfering" small cells will somehow hurt the network performance. On the other hand, lightly loaded cells will not provide a major capacity increase, since the load is light. If the loads on each tier are the same, then the coverage probability neither increases or decreases as tiers or BSs are added, and the sum throughput increases linearly with the number of BSs and/or tiers.

\section{SYSTEM MODEL}

We model a downlink $\mathrm{HCN}$ with $K$ classes (or tiers) of BSs; we denote the set $\{1,2, \ldots K\}$ by $\mathcal{K}$. BSs of the $i^{\text {th }}$ class transmit with power $P_{i}$, have a target Signal-toInterference-plus-Noise-Ratio (SINR) of $\beta_{i}$ and are assumed to form a realization of an independent homogeneous Poisson Point Process (PPP) $\Phi_{i}$ with density $\lambda_{i}$. This model has been validated vs. a real world deployment for macrocells $(K=1)$ in [3]. The model is likely even more sensible for $K$-tier HCNs due to the increased uncertainty ("randomness") in the deployment of smaller cells, and as noted in the introduction has been adopted by academia and industry alike in the past year. 
Without loss of generality, we perform analysis on a typical mobile user located at origin and we consider the max-SINR, equivalently max-power, connectivity model. In closed access - considered later - the mobile connects to the strongest BS in the allowed subset $\mathcal{B} \subseteq \mathcal{K}$ of tiers. Since HCNs are typically interference-limited [12], we ignore thermal noise for notational simplicity, but this is not essential. The wireless channel follows standard distance based path loss with exponent $\alpha$ along with Rayleigh fading. Hence the received power at a typical mobile from a BS located at point $x \in \Phi_{i}$ can be expressed as $P_{i} h_{x}\|x\|^{-\alpha}$, where $h_{x} \sim \exp (1)$ and $\|x\|^{-\alpha}$ is the distance based path loss. Assuming $\mathcal{Z}_{i}$ to be the set of $i^{\text {th }}$-tier interfering BSs (possibly thinned version of $\Phi_{i}$ ), the downlink SIR at the typical mobile user when it connects to the BS located at point $y \in \Phi_{i}$ is:

$$
\operatorname{SIR}(y)=\frac{P_{i} h_{y}\|y\|^{-\alpha}}{\sum_{k=1}^{K} \sum_{x \in \cup \mathcal{Z}_{i}} P_{k} h_{x}\|x\|^{-\alpha}} .
$$

\section{A. Proposed Load Model and Mathematical Preliminaries}

Network "load" is modeled as the likelihood of transmission by a given $\mathrm{BS}$ at a randomly chosen time instant. This can also be visualized as the BS activity factor, formally defined as the fraction of time for which a BS transmits. Correlation in loads across time and space are ignored.

Thus, we assume that a typical mobile connects to the strongest BS and conditioned on this connection, the interferer belonging to the $i^{\text {th }}$ tier transmits independently with a probability $p_{i}$ and is idle with a probability $1-p_{i}$. This conditioning makes it harder to analyze this system model since we do not have a priori knowledge about the serving BS and hence it is not possible to isolate the interference field. To overcome this, we partition each tier $\Phi_{m}$ independently into two sets of BSs $\Psi_{m}$ and $\Delta_{m}$, where $\Psi_{m}$ and $\Delta_{m}$ are both independent PPPs with densities $p_{m} \lambda_{m}$ and $\left(1-p_{m}\right) \lambda_{m}$. The set $\Psi_{m}$ represents the set of active BSs of tier $m$ with the possibility of one of them being a serving BS, and $\Delta_{m}$ represents the set of idle BSs of tier $m$ with an exception that it could also contain the serving BS since partitioning was done independently. The advantage of this partitioning is that the interferers are confined to the set $\Psi=\bigcup_{m \in \mathcal{K}} \Psi_{m}$.

For ease of notation, we define the maximum signal strength from a set of nodes $\mathcal{A}$ as $M(\mathcal{A})=\sup _{x \in \mathcal{A}} P_{\mathcal{A}} h_{x}\|x\|^{-\alpha}$ and the total received power at the origin from the set of active BSs as $I=\sum_{i=1}^{K} \sum_{x \in \Psi_{i}} P_{i} h_{x}\|x\|^{-\alpha}$, which denotes the net interference power if $\Psi$ does not include the serving BS and the interference plus signal power if it includes the serving BS. From the definition of $M\left(\Psi_{i}\right)$ and $I$, it is easy to see that $\mathbf{1}\left(\frac{M\left(\Psi_{i}\right)}{I-M(\Psi)}<\beta_{i}\right)=1$ only if no active BS in the set $\Psi_{i}$ can connect to the mobile. Similarly, $\mathbf{1}\left(\frac{M\left(\Delta_{i}\right)}{I}<\beta_{i}\right)=1$ only if no $\mathrm{BS}$ in the set $\Delta_{i}$ is able to connect to the mobile. The second event is defined to cover the possibility that a serving BS may lie in the set $\Delta_{i}$. Recalling that a mobile is in outage (not in coverage) if none of the BSs in the whole network provides SIR that is greater than the corresponding target for that tier, the coverage probability can now be defined in terms of these two events as:

$$
\mathrm{P}_{\mathrm{c}}=1-\mathbb{E}\left[\prod_{i \in \mathcal{K}} \mathbf{1}\left(\frac{M\left(\Psi_{i}\right)}{I-M(\Psi)}<\beta_{i}\right) \mathbf{1}\left(\frac{M\left(\Delta_{i}\right)}{I}<\beta_{i}\right)\right] \text {. }
$$

For tractability, we assume that the target-SIRs $\beta_{i}$ are greater than $0 \mathrm{~dB}$, i.e., $\beta_{i}>1, \forall i$. This is in fact the case for a large fraction of mobile users and only a few edge users might violate this assumption. Moreover, in [6], we have shown that the results derived under this weaker assumption hold down until around $-4 \mathrm{~dB}$ which covers a large fraction of cell edge users as well. The reason why this assumption is helpful is because it ensures that at most one BS in the active set $\Psi$ meets the target SIR requirements for a typical mobile user. Refer to [6] for a detailed discussion on this assumption and its application in coverage analysis of a fully-loaded $K$-tier $\mathrm{HCN}$.

\section{B. Coverage Regions}

To understand the effect of proposed model on the coverage footprints of various BSs, consider a realization of a three tier $\mathrm{HCN}$ shown in Fig. 1. In the left figure, we plot the coverage regions assuming a fully loaded network by tessellating the space according to max-SIR connectivity model. Clearly, this plot does not resemble a classical Voronoi tessellation due to the differences in the transmit powers of BSs across tiers. Moreover, it should be noted that the "cell edges" are not as sharp in reality due to fading and shadowing, which are averaged out for these illustrative plots. The effect of incorporating the proposed load model can be understood in two equivalent ways: i) thinning of the interference field (middle figure), ii) biasing of a typical mobile towards its serving BS (right figure). While the former is a direct result of conditional thinning, the latter is an indirect consequence of the expansion of coverage regions in the thinned interference field.

\section{Coverage Probability}

This is the main technical section of this paper where we derive the probability that a typical mobile is in coverage. We first derive coverage probability for an open access network, where the mobile is allowed to connect to any BS in the network. The result for the closed access network or the closed subscriber group, where the mobile is restricted to connect only to the $\mathcal{B} \subseteq \mathcal{K}$ tiers, immediately follow.

\section{A. Exact Expression for Coverage Probability}

We start by stating the Laplace transform of $I$, i.e., $\mathcal{L}_{I}(s)=$ $\mathbb{E}[\exp (-s I)]$, in Lemma 1 , which will be useful in the derivation of coverage probability. The proof is given in [6].

Lemma 1. The Laplace transform of I can be expressed as:

$$
\mathcal{L}_{I}(s)=\exp \left(-s^{2 / \alpha} C(\alpha) \sum_{l=1}^{K} p_{l} \lambda_{l} P_{l}^{2 / \alpha}\right),
$$

where $C(\alpha)$ is given by $C(\alpha)=\frac{2 \pi^{2} \csc \left(\frac{2 \pi}{\alpha}\right)}{\alpha}$. 

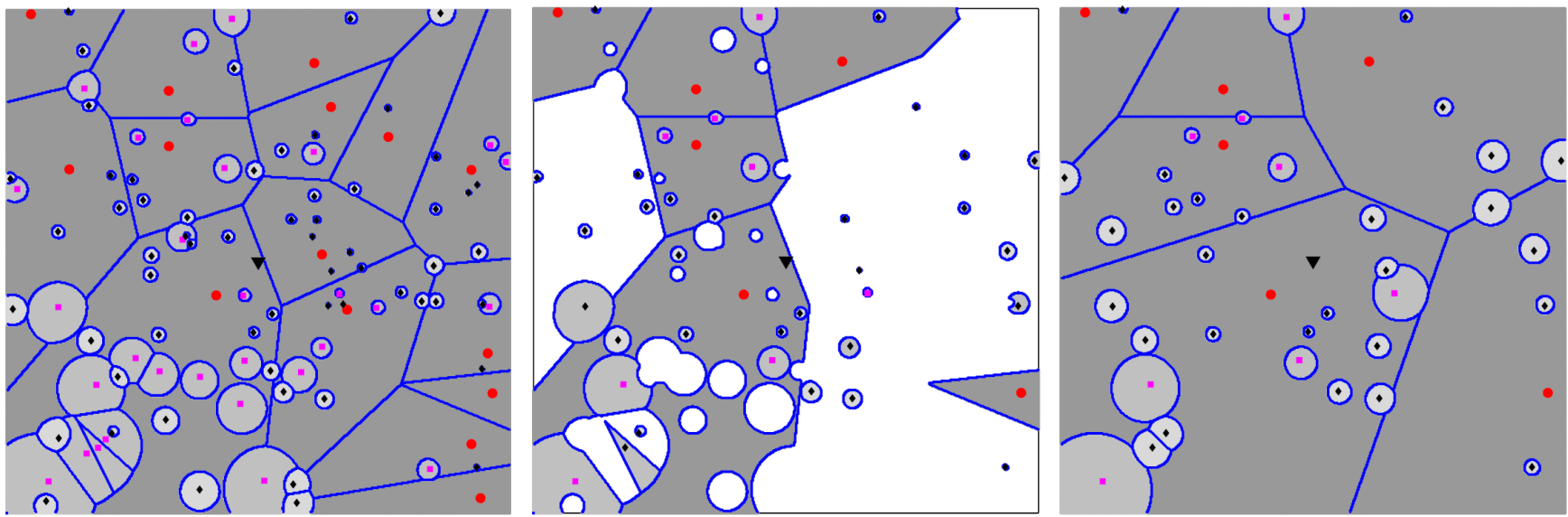

Fig. 1. Coverage regions in a realization of a three-tier network with $\lambda_{2}=2 \lambda_{1}, \lambda_{3}=4 \lambda_{1}, P_{1}=100 P_{2}$ and $P_{1}=1000 P_{3}$. The big circles represent macrocells, squares represent picocells, small diamonds represent femtocells and big triangle represents typical mobile. The left figure depicts fully loaded system and the other two have the interference field thinned by $p_{1}=.6$ and $p_{2}=p_{3}=.4$. To highlight the removal of certain interferers, their original coverage regions are removed as well in the middle figure. The coverage regions in the right figure are redrawn based only on the active set of interferers. It highlights that the typical mobile is now "biased" towards its serving BS and the new coverage regions are enlarged due to thinning of the interference field.

The following Lemma deals with fractional moments of interference and is the main technical result required for evaluating the coverage probability for this model.

Lemma 2. Let $\Psi_{i}$ denote the set of active transmitters of tier $i$ and $\delta_{i}=\beta_{i} /\left(1+\beta_{i}\right)$. Let I denote the total received power from the BSs in the set $\Psi$ and for notational simplicity define $\mathcal{T}=\mathbf{1}\left(\max _{i \in \mathcal{K}} \frac{M\left(\Psi_{i}\right)}{\delta_{i}}<I\right) I^{-2 / \alpha}$. Then

$$
\mathbb{E}\left[\mathcal{T}^{m}\right]=\frac{m ! g(m)}{(-A)^{m}}
$$

where

$$
g(m)=\left(\frac{-A}{\eta}\right)^{m}\left\{\frac{1}{\Gamma\left(1+\frac{2 m}{\alpha}\right)}-\frac{B}{\eta} \frac{\pi \Gamma\left(1+\frac{2}{\alpha}\right)}{\Gamma\left(1+\frac{(m+1) 2}{\alpha}\right)}\right\},
$$

and

$$
\begin{aligned}
A & =\pi \Gamma(1+2 / \alpha) \sum_{l \in \mathcal{K}}\left(1-p_{l}\right) \lambda_{l} P_{l}^{2 / \alpha} \beta_{l}^{-2 / \alpha} \\
B & =\sum_{i \in \mathcal{K}} \frac{\lambda_{i} p_{i} P_{i}^{\frac{2}{\alpha}} \beta_{i}^{-\frac{2}{\alpha}}{ }_{2} F_{1}\left(1, \frac{2 m}{\alpha}, 1+\frac{(m+1) 2}{\alpha}, \frac{1}{1+\beta_{i}}\right)}{\left(1+\beta_{i}\right)^{2 m / \alpha}} \\
\eta & =C(\alpha) \sum_{l=1}^{K} p_{l} \lambda_{l} P_{l}^{2 / \alpha}
\end{aligned}
$$

The hypergeometric function is denoted by ${ }_{2} F_{1}(a, b, c, z)=$ $\frac{\Gamma(c)}{\Gamma(b) \Gamma(c-b)} \int_{0}^{1} \frac{t^{b-1}(1-t)^{c-b-1}}{(1-t z)^{a}} \mathrm{~d} t$.

Proof: See Appendix A.

Using these Lemmas, we now derive the main result which characterizes the coverage probability of a typical mobile in the network.

Theorem 1 (Open Access). The downlink coverage probability for a typical mobile user in a $K$-tier open access network assuming $\beta_{i}>1, \forall i$, is

$$
\mathrm{P}_{\mathrm{c}}=\frac{\pi}{C(\alpha)} \frac{\sum_{i \in \mathcal{K}} p_{i} \lambda_{i} P_{i}^{2 / \alpha} \beta_{i}^{-2 / \alpha}}{\sum_{i=1}^{K} p_{i} \lambda_{i} P_{i}^{2 / \alpha}}-\sum_{m=1}^{\infty} g(m),
$$

Proof: The coverage probability is given by (2). Since the point processes $\Delta_{i}$ and the corresponding fading random variables are independent, conditioning on the common interference, we can move the expectation inside the product. Hence

$1-\mathrm{P}_{\mathrm{c}}=\mathbb{E}\left[\prod_{i=1}^{K} \mathbf{1}\left(\frac{M\left(\Psi_{i}\right)}{I-M(\Psi)}<\beta_{i}\right) \mathbb{E}\left[\mathbf{1}\left(M\left(\Delta_{i}\right)<\beta_{i} I\right)\right]\right]$,

where the inner expectation is with respect to the inactive transmitter sets. The inner expectation can be simplified to:

$$
\begin{aligned}
& \mathbb{E}\left[\prod_{x \in \Delta_{i}} \mathbf{1}\left(P_{i} h\|x\|^{-\alpha}<\beta_{i} I\right)\right] \\
& \stackrel{(a)}{=} \mathbb{E}\left[\prod_{x \in \Delta_{i}}\left(1-\exp \left(-\beta_{i} P_{i}^{-1} I\|x\|^{\alpha}\right)\right)\right] \\
& \stackrel{(b)}{=} \exp \left(-\left(1-p_{i}\right) \lambda_{i} \int_{x \in \mathbb{R}^{2}} e^{-\beta_{i} P_{i}^{-1} I\|x\|^{\alpha}} \mathrm{d} x\right) \\
& \stackrel{(c)}{=} \exp \left(-\left(1-p_{i}\right) \lambda_{i} \beta_{i}^{-\frac{2}{\alpha}} I^{-\frac{2}{\alpha}} P_{i}^{\frac{2}{\alpha}} \pi \Gamma\left(1+\frac{2}{\alpha}\right)\right),
\end{aligned}
$$

where $(a)$ follows form the fact that fading is Rayleigh distributed, i.e., $h \sim \exp (1),(b)$ follows from the probability generating functional (PGFL) of PPP [13] and (c) follows from some algebraic manipulations to reduce the integral to a Gamma function. Now recalling the expression of $A$ given by (5), we can write:

$$
1-\mathrm{P}_{\mathrm{c}}=\mathbb{E}\left[\mathbf{1}\left(\max _{i \in \mathcal{K}} \frac{M\left(\Psi_{i}\right)}{\delta_{i}}<I\right) \exp \left(-A I^{-2 / \alpha}\right)\right] .
$$

Using the Taylor series expansion of $\exp (-x)$, exchanging the infinite summation and expectation,

$$
1-\mathrm{P}_{\mathrm{c}}=\sum_{m=0}^{\infty} \frac{(-A)^{m}}{m !} \mathbb{E}\left[\mathbf{1}\left(\max _{i \in \mathcal{K}} \frac{M\left(\Psi_{i}\right)}{\delta_{i}}<I\right) I^{-2 m / \alpha}\right] .
$$


The summation can be split as

$$
1-\mathrm{P}_{\mathrm{c}}=\mathbb{P}\left(\max _{i \in \mathcal{K}} \frac{M\left(\Psi_{i}\right)}{\delta_{i}}<I\right)+\sum_{m=1}^{\infty} \frac{(-A)^{m}}{m !} \mathbb{E}\left[\mathcal{T}^{m}\right]
$$

The term $1-\mathbb{P}\left(\max _{i \in \mathcal{K}} \frac{M\left(\Psi_{i}\right)}{\delta_{i}}<I\right)$ is the coverage probability in a fully loaded heterogeneous network where the $m$-th tier density is $p_{m} \lambda_{m}$. This is derived in [6] and is given by:

$$
1-\mathbb{P}\left(\max _{i} \frac{M\left(\Psi_{i}\right)}{\delta_{i}}<I\right)=\frac{\pi}{C(\alpha)} \frac{\sum_{i=1}^{K} p_{i} \lambda_{i} P_{i}^{\frac{2}{\alpha}} \beta_{i}^{-\frac{2}{\alpha}}}{\sum_{i=1}^{K} p_{i} \lambda_{i} P_{i}^{\frac{2}{\alpha}}} .
$$

Using Lemma 2 to evaluate $\mathbb{E}\left[\mathcal{T}^{m}\right]$, we obtain the result.

We note that the expression of coverage probability involves infinite summation over the sequence $g(m)$. Therefore, we first show that the infinite summation converges by showing that $|g(m)| \rightarrow 0$ as $m \rightarrow \infty$. Observe that:

$$
\begin{aligned}
|g(m)| & \leq\left(\frac{A}{\eta}\right)^{m} \frac{1}{\Gamma\left(1+\frac{2 m}{\alpha}\right)} \leq \frac{(A / \eta)^{m}}{\left\lfloor 1+\frac{2 m}{\alpha}\right\rfloor !}=\frac{(A / \eta)^{m}}{\left\lceil\frac{2 m}{\alpha}\right\rceil !} \\
& =\frac{\left[(A / \eta)^{\frac{m}{\left\lceil\frac{2 m}{\alpha}\right\rceil}}\right]^{\left\lceil\frac{2 m}{\alpha}\right\rceil}}{\left\lceil\frac{2 m}{\alpha}\right\rceil !} \rightarrow 0,
\end{aligned}
$$

where the limiting argument follows from the fact that the sequence of the form $x^{n} / n ! \rightarrow 0$. In addition to proving that the series converges, this upper bound on $|g(m)|$ also sheds light on the behavior of the sequence $g(m)$. If $A / \eta<1$, the bound decreases monotonously with $m$ and hence it is enough to consider only a few significant terms to closely approximate the infinite sum. However, if $A / \eta>1$, especially if $A / \eta \gg 1$, the upper bound first increases until $\left\lceil\frac{2 m}{\alpha}\right\rceil \leq(A / \eta)^{\frac{2 m}{\left\lceil\frac{2 m}{\alpha}\right\rceil}}$ and decreases thereafter. Therefore, the number of significant terms of $g(m)$ required to approximate the infinite sum would be relatively higher in this case.

We now provide the exact expression for the coverage probability in a closed access network in the following Theorem. We recall that that coverage probability in closed-access is given by (2) with the only change that the product is over $\mathcal{B}$ instead of $\mathcal{K}$. By definition, coverage probability in closed access is less than that of open access. Using this definition, the proof proceeds exactly same as that of Theorem 1, and hence is not provided.

Theorem 2 (Closed Access). The downlink coverage probability of a typical mobile in a K-tier closed access network where a mobile is allowed to connect to $\mathcal{B} \subseteq \mathcal{K}$ tiers assuming $\beta_{i}>1, \forall i$, is

$$
\mathrm{P}_{\mathrm{c}}=\frac{\pi}{C(\alpha)} \frac{\sum_{i \in \mathcal{B}} p_{i} \lambda_{i} P_{i}^{2 / \alpha} \beta_{i}^{-2 / \alpha}}{\sum_{i=1}^{K} p_{i} \lambda_{i} P_{i}^{2 / \alpha}}-\sum_{m=1}^{\infty} g_{c}(m),
$$

where $g_{c}(m)$ and corresponding $A$ and $B$ are given by (4), (5) and (6), respectively, with the only difference that the summations defined over set $\mathcal{K}$ are now over set $\mathcal{B}$.

\section{B. Special Cases of Interest}

We now use the results derived in this section to study some special cases and compare the system performance with already known results for fully-loaded system. First, we note that for a fully-loaded system, the value of $A=0$ and hence $g(m)=g_{c}(m)=0, \forall m$. Therefore, the coverage probability in this case can be expressed as:

$$
\mathrm{P}_{\mathrm{c}}=\frac{\pi}{C(\alpha)} \frac{\sum_{i \in \mathcal{K}} \lambda_{i} P_{i}^{2 / \alpha} \beta_{i}^{-2 / \alpha}}{\sum_{i=1}^{K} \lambda_{i} P_{i}^{2 / \alpha}},
$$

which is the same as the Corollary 1 in [6]. The coverage probability in closed access is also given by the same expression with the only difference that the summation over the set $\mathcal{K}$ is now over the set $\mathcal{B}$.

For a single-tier open-access network, the coverage probability derived in Theorem 1 can be simplified and is expressed as the following Corollary.

Corollary 1 (Single-Tier). The coverage probability for the single tier open access network with BS activity factor $p$ is

$$
\mathrm{P}_{\mathrm{c}}=\frac{\pi \beta^{-2 / \alpha}}{C(\alpha)}-\sum_{m=1}^{\infty} g(m),
$$

where the terms $A / \eta$ and $B / \eta$ appearing in $g(m)$ are

$$
\begin{aligned}
& \frac{A}{\eta}=\frac{\pi \Gamma\left(1+\frac{2}{\alpha}\right)(1-p)}{C(\alpha) p \beta^{2 / \alpha}} \\
& \frac{B}{\eta}=\frac{{ }_{2} F_{1}\left(1, \frac{2 m}{\alpha}, 1+\frac{(m+1) 2}{\alpha}, \frac{1}{1+\beta}\right)}{C(\alpha) \beta^{2 / \alpha}(1+\beta)^{2 m / \alpha}} .
\end{aligned}
$$

Remark 1 (Scale-invariance of a single-tier network). From Corollary 1, we note that for any BS activity factor $p$, the coverage probability in a single-tier open-access network is independent of the BS density $\lambda$ and transmit power P. This is henceforth referred to as "scale-invariance" of cellular networks to changes in the BS density and their transmit powers.

Remark 1 is a generalization of a similar result derived for fully-loaded networks in [6], which can easily be seen from (18). In addition to single-tier networks, (18) also shows that the general fully-loaded open-access multi-tier networks also exhibit scale-invariance if the target SIRs for all the tiers are the same. Motivated by this observation, we study the coverage probability for our proposed load model in open-access multitier networks under the assumption that the target SIR is the same for all tiers in the next Corollary.

Corollary 2 (Coverage Probability: $K$-Tier with same $\beta$ ). The coverage probability for a K-tier open-access network assuming target SIRs to be the same $(=\beta)$ for all the tiers is given by (19), with the difference that $A / \eta$ appearing in $g(m)$ is now defined as:

$$
\frac{A}{\eta}=\frac{\pi \Gamma\left(1+\frac{2}{\alpha}\right)}{C(\alpha) \beta^{2 / \alpha}} \frac{\sum_{l=1}^{K}\left(1-p_{l}\right) \lambda_{l} P_{l}^{2 / \alpha}}{\sum_{l=1}^{K} p_{l} \lambda_{l} P_{l}^{2 / \alpha}},
$$

and $B / \eta$ is given by (21). 


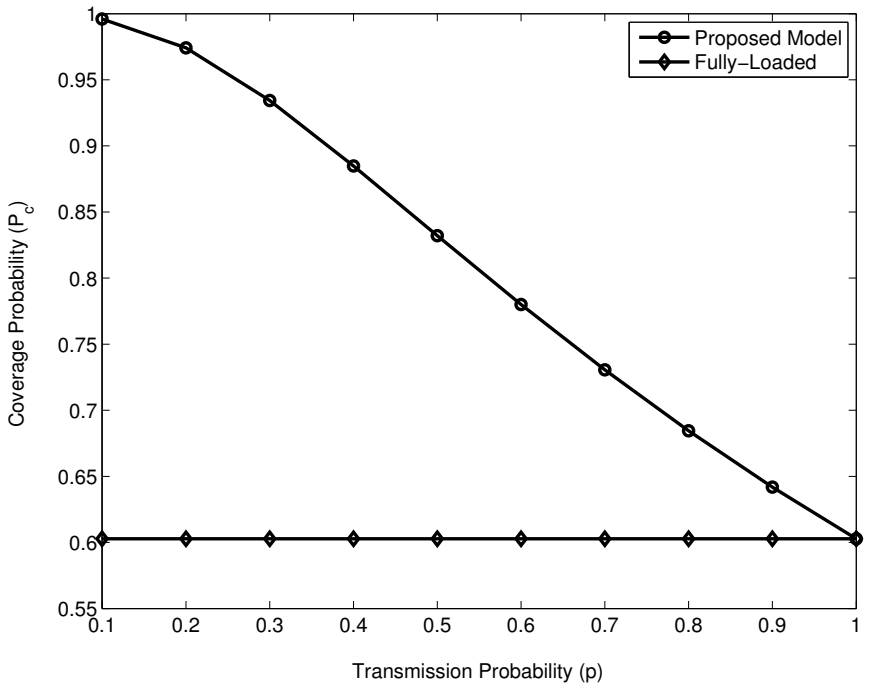

Fig. 2. Coverage probability as a function of transmission probability is a single tier network $(\beta=1$ and $\alpha=3.8)$.

Remark 2 (Scale-Invariance of $K$-tier HCNs with same $\beta$ ). From Corollary 2, we note that the coverage probability for $K$ tier HCNs is not scale-invariant in general, even when target SIRs of all the tiers are the same. However, the invariance property does hold when the BS activity factors of all the tiers are the same. Interestingly, the coverage probability in this case is the same as that of a single-tier network given by Corollary 1.

To understand this remark, we consider the following simple example.

Example 1 (Scale-invariance in a 2-tier HCN). Consider a two-tier network with $B S$ activity factors $p_{1}$ and $p_{2}$. If $p_{1}<p_{2}$, increasing the density of the first tier leads to a higher increase in the intended power due to the higher likelihood of having a closer tier-1 BS as the serving BS but a relatively smaller increase in the interference power. The coverage probability in this case is expected to increase. On the other hand, if $p_{1}>p_{2}$, increasing the density of tier-1 BSs leads to higher increase in the interference power as compared to the intended power, leading to a decrease in the coverage probability. The two effects cancel each other when the activity factors of the two tiers are the same.

\section{NumericAl Results}

Since most of the analytical results derived in this paper are fairly self-explanatory and do not require separate numerical treatment, we will provide only those results which help better visualize certain important trends.

First, we compare the coverage results with those of a fullyloaded system in Fig. 2. Although a huge difference in the coverage guarantees was expected for very low BS activity factors, it is indeed interesting that the coverage estimates assuming full load are quite pessimistic even for reasonably high load scenarios, such as $p=.7-.8$.

To study scale invariance and the effect of adding small cells, we now consider a two-tier system and plot the coverage

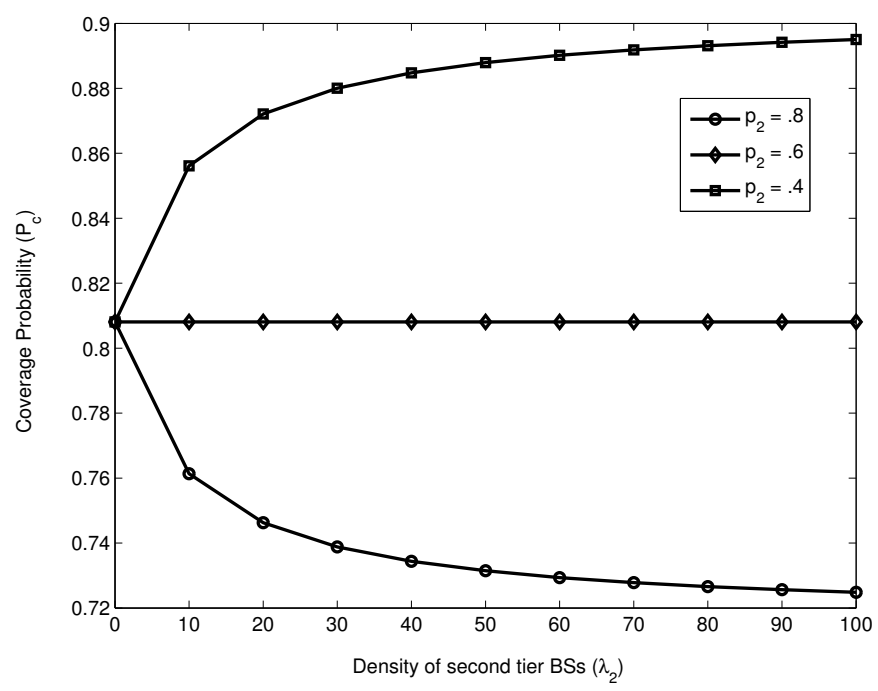

Fig. 3. Coverage probability in a two-tier network as a function of $\lambda_{2}$ $\left(\beta=[1,1], P=[1, .01], \lambda_{1}=1, p_{1}=.6\right.$ and $\left.\alpha=4\right)$.

probability as a function of the density of second tier for various BS activity factors in Fig. 3. The target SIR is considered to be the same for both the tiers. We first note that the network is invariant to the changes in density when $p_{1}=p_{2}$ as discussed in the last section. More importantly, we note that the coverage probability increases with $\lambda_{2}$ when the second tier BSs are less active than the first tier. This is a possibly important result from the perspective of small-cells, which are generally less active than macrocell BSs. Therefore, the coverage probability of the network should increase with the addition of small-cells in this regime. On the other hand, if a tier of BSs is added which is more active than the macrocells, the coverage would decrease, although this case seems pretty unlikely given the high load handled by the macrocells.

\section{Conclusions}

In this paper, we have developed a tractable load model for $K$-tier HCNs by defining a notion of conditional-thinning of interference, conditional on the connection of a typical mobile to its serving BS. Using tools from stochastic geometry and point process theory, we derived a simple expression for the average coverage probability of a typical mobile. Apart from other design insights, our analysis shows that the addition of small cells to macrocellular networks will increase the overall coverage probability of the network and hence provides a strong rebuttal to the viewpoint that unplanned infrastructure might bring down a cellular network due to increased interference.

\section{APPENDIX A}

PROOF OF LEMMA 2:

Being consistent with the definition of $\mathcal{T}$, we note that:

$$
\mathcal{T}^{m}=\mathbf{1}\left(\max _{i \in \mathcal{K}} \frac{M\left(\Psi_{i}\right)}{\delta_{i}}<I\right) I^{-2 m / \alpha} .
$$


To proceed with the proof, we represent $I^{-2 m / \alpha}$ in terms of $\Gamma(x)$ as:

$$
I^{-2 m / \alpha}=\frac{1}{\Gamma(2 m / \alpha)} \int_{0}^{\infty} e^{-s I} s^{-1+\frac{2 m}{\alpha}} \mathrm{d} s, m \geq 1,
$$

where $\Gamma(x)$ is the standard gamma function. Using this representation of $I^{-2 m / \alpha}$ followed by exchanging the order of expectation and integral, we can express $\mathbb{E}\left[\mathcal{T}^{m}\right]$ as:

$$
\frac{1}{\Gamma(2 m / \alpha)} \int_{0}^{\infty} s^{-1+\frac{2 m}{\alpha}} \mathbb{E}\left[e^{-s I} \mathbf{1}\left(\max _{i \in \mathcal{K}} \frac{M\left(\Psi_{i}\right)}{\delta_{i}}<I\right)\right] \mathrm{d} s .
$$

Under the assumption $\beta_{i}>1, \forall i$, we know that only one BS in the whole network can establish a downlink connection with a typical mobile. Hence,

$$
\mathbf{1}\left(\max _{i \in \mathcal{K}} \frac{M\left(\Psi_{i}\right)}{\delta_{i}}>I\right)=\sum_{i=1}^{K} \sum_{x \in \Psi_{i}} \mathbf{1}\left(\operatorname{SIR}(x)>\beta_{i}\right),
$$

where $\operatorname{SIR}(x)$ is the received $\operatorname{SIR}$ when a typical mobile is associated with the BS located at $x \in \Psi_{i}$. Using this expression, the expectation term of (25) can be written as:

$$
\begin{aligned}
& \mathbb{E}\left[e^{-s I} \mathbf{1}\left(\max _{i} \frac{M\left(\Psi_{i}\right)}{\delta_{i}}<I\right)\right] \\
& =\mathbb{E}\left[e^{-s I}\right]-\sum_{i=1}^{K} \mathbb{E}\left[e^{-s I} \sum_{x \in \Psi_{i}} \mathbf{1}\left(\operatorname{SIR}(x)>\beta_{i}\right)\right] .
\end{aligned}
$$

From Lemma 1, we know the Laplace transform of total interference and hence the first term in the above expression can be directly written as:

$$
\mathbb{E}\left[e^{-s I}\right]=\exp ^{-s^{2 / \alpha} C(\alpha) \sum_{l=1}^{K} p_{l} \lambda_{l} P_{l}^{2 / \alpha}} .
$$

To evaluate the expectation in the second term of (27), we first denote the effective interference as $I^{\prime}=I-P_{i} h_{x}\|x\|^{-\alpha}$ and note that the Laplace transforms of $I$ and $I^{\prime}$ are the same. The expectation can now be simplified as:

$$
\begin{aligned}
& \mathbb{E}\left[e^{-s I} \sum_{x \in \Psi_{i}} \mathbf{1}\left(\operatorname{SIR}(x)>\beta_{i}\right)\right] \\
& =\mathbb{E}\left[\sum_{x \in \Psi_{i}} \exp \left(-s I^{\prime}+P_{i} h_{x}\|x\|^{-\alpha}\right) \mathbf{1}\left(\frac{P_{i} h_{x}\|x\|^{-\alpha}}{I^{\prime}}>\beta_{i}\right)\right]
\end{aligned}
$$

$\stackrel{(a)}{=} \mathbb{E}\left[\sum_{x \in \Psi_{i}} e^{-s I^{\prime}} \mathbb{E}_{h_{x}}\left[e^{-P_{i} h_{x}\|x\|^{-\alpha}} \mathbf{1}\left(h_{x}>\beta_{i} I^{\prime} P_{i}^{-1}\|x\|^{\alpha}\right)\right]\right]$

$$
\stackrel{(b)}{=} \mathbb{E}\left[\sum_{x \in \Psi_{i}} \frac{\mathbb{E}_{I^{\prime}}\left[\exp \left(-I^{\prime}\left(s\left(1+\beta_{i}\right)+\beta_{i} P_{i}^{-1}\|x\|^{\alpha}\right)\right)\right]}{1+s P_{i}\|x\|^{-\alpha}}\right],
$$

where $(a)$ follows from the fact that fading is independent of all the other random variables and $(b)$ follows from the fact that $h_{x} \sim \exp (1)$. Now, using the Laplace transform of $I^{\prime}$ and recalling $\eta=\sum_{l=1}^{K} \lambda_{l} p_{l} P_{l}^{2 / \alpha} C(\alpha)$, it can be further simplified to:

$$
\mathbb{E}\left[\sum_{x \in \Psi_{i}} \frac{e^{-\eta\left(s\left(1+\beta_{i}\right)+\beta_{i} P_{i}^{-1}\|x\|^{\alpha}\right)^{2 / \alpha}}}{1+s P_{i}\|x\|^{-\alpha}}\right],
$$

and using Campbell Mecke theorem [13] to:

$$
\lambda_{i} p_{i} \int_{\mathbb{R}^{2}} \frac{e^{-\eta\left(s\left(1+\beta_{i}\right)+\beta_{i} P_{i}^{-1}\|x\|^{\alpha}\right)^{2 / \alpha}}}{1+s P_{i}\|x\|^{-\alpha}} \mathrm{d} x .
$$

With this we have now simplified both the terms of (27) given respectively by (28) and (33). We now substitute the first term in (25) and evaluate the integral with respect to $s$ as:

$$
\int_{0}^{\infty} s^{-1+2 m / \alpha} e^{-\eta s^{2 / \alpha}} \mathrm{d} s=\frac{\eta^{-m} \alpha(m-1) !}{2},
$$

where the solution follows from the substitution $s^{2 / \alpha} \rightarrow y$ followed by integration by parts. Now substituting the second term (given by (33)) in (25), we get the following integral:

$$
\lambda_{i} p_{i} \int_{0}^{\infty} \int_{\mathbb{R}^{2}} \frac{s^{-1+2 m / \alpha} e^{-\eta\left(s\left(1+\beta_{i}\right)+\beta_{i} P_{i}^{-1}\|x\|^{\alpha}\right)^{2 / \alpha}}}{1+s P_{i}\|x\|^{-\alpha}} \mathrm{d} x \mathrm{~d} s,
$$

which can be simplified to:

$$
\begin{aligned}
& \frac{\lambda_{i} p_{i} P_{i}^{2 / \alpha} \eta^{-(m+1)} m ! \pi \beta_{i}^{-2 / \alpha}}{\left(1+\beta_{i}\right)^{2 m / \alpha}} \frac{\Gamma(2 m / \alpha) \Gamma(1+2 / \alpha)}{\Gamma(1+(m+1) 2 / \alpha)} \\
& { }_{2} F_{1}\left(1,2 m / \alpha, 1+(m+1) 2 / \alpha,\left(1+\beta_{i}\right)^{-1}\right)
\end{aligned}
$$

where ${ }_{2} F_{1}$ is the generalized hypergeometric function. Combining all the above we obtain the result.

\section{REFERENCES}

[1] Cisco, "Cisco visual networking index: Global mobile data traffic forecast update, 2011 - 2016," white paper, Feb. 2012.

[2] Qualcomm, "LTE advanced: heterogeneous networks," white paper, Jan. 2011.

[3] J. G. Andrews, F. Baccelli, and R. K. Ganti, "A tractable approach to coverage and rate in cellular networks," IEEE Trans. on Communications, vol. 59, no. 11, pp. 3122-3134, Nov. 2011.

[4] F. Baccelli and B. Blaszczyszyn, Stochastic Geometry and Wireless Networks. NOW: Foundations and Trends in Networking, 2009.

[5] H. S. Dhillon, R. K. Ganti, and J. G. Andrews, "A tractable framework for coverage and outage in heterogeneous cellular networks," in Proc., Information Theory and its Applications (ITA), San Diego, CA, Feb. 2011.

[6] H. S. Dhillon, R. K. Ganti, F. Baccelli, and J. G. Andrews, "Modeling and analysis of K-tier downlink heterogeneous cellular networks," IEEE Journal on Sel. Areas in Communications, vol. 30, no. 3, pp. 550 - 560, Apr. 2012.

[7] S. Mukherjee, "Distribution of downlink SINR in heterogeneous cellular networks," IEEE Journal on Sel. Areas in Communications, vol. 30 , no. 3, pp. 575 - 585, Apr. 2012.

[8] H.-S. Jo, Y. J. Sang, P. Xia, and J. G. Andrews, "Heterogeneous cellular networks with flexible cell association: A comprehensive downlink SINR analysis," Jul. 2011, submitted to IEEE Trans. on Wireless Communications. Available Online: arxiv.org/abs/1107.3602.

[9] H. S. Dhillon, R. K. Ganti, F. Baccelli, and J. G. Andrews, "Coverage and ergodic rate in K-tier downlink heterogeneous cellular networks," in Proc., Allerton Conf. on Comm., Control, and Computing, Monticello, IL, Sep. 2011.

[10] A. Damnjanovic, J. Montojo, Y. Wei, T. Ji, T. Luo, M. Vajapeyam, T. Yoo, O. Song, and D. Malladi, "A survey on 3GPP heterogeneous networks," IEEE Wireless Communications Magazine, vol. 18, no. 3, pp. 10 - 21, Jun. 2011.

[11] A. Ghosh, J. G. Andrews, N. Mangalvedhe, R. Ratasuk, B. Mondal, M. Cudak, E. Visotsky, T. A. Thomas, P. Xia, H. S. Jo, H. S. Dhillon, and T. D. Novlan, "Heterogeneous cellular networks: From theory to practice," IEEE Communications Magazine, Jun. 2012.

[12] G. Boudreau, J. Panicker, N. Guo, R. Chang, N. Wang, and S. Vrzic, "Interference coordination and cancellation for 4G networks," IEEE Communications Magazine, vol. 47, no. 4, pp. 74 - 81, Apr. 2009.

[13] D. Stoyan, W. S. Kendall, and J. Mecke, Stochastic Geometry and Its Applications, 2nd ed. Chichester: John Wiley and Sons, 1995. 